\title{
Epithelioid Sarcoma NCI Grade 3
}

National Cancer Institute

\section{Source}

National Cancer Institute. Epithelioid Sarcoma NCI Grade 3. NCI Thesaurus. Code C9410.

An epithelioid sarcoma with more than 15\% necrosis. 